\title{
User-Customisable Policy Monitoring for Multi-tenant Cloud Architectures
}

\author{
Ming-Xue Wang and Claus Pahl \\ School of Computing, Dublin City University \\ Dublin, Ireland \\ [mwang | cpahl] @computing.dcu.ie
}

\begin{abstract}
Cloud computing needs end-user customisation and personalisation of multi-tenant cloud service offerings. Particularly, QoS and governance policy management and monitoring is needed. We propose a user-customisable policy definition solution that can be enforced in multitenant cloud offerings through automated instrumentation and monitoring. Service processes run by cloud and SaaS providers can be made policy-aware in a transparent way.
\end{abstract}

Key words: Cloud architecture; Policy customisation; Monitoring.

\section{Introduction}

Cloud customers should to be allowed to better control and customise cloud offerings through specific management interfaces. This is a multi-tenancy environment where users have varying requirements [1]. Governance and QoS issues are concerns usually split between provider and user. This requires a monitoring and enforcement platform, where these policies can be configured. Two key objectives of our policy definition, instrumentation and monitoring solution can be singled out. Firstly, user-configured policy management for multi-tenancy allows end-user customisable cloud computing. Secondly, our process-level policy management works not only for service-level offerings, but also for process-level architectures, where the provider implements an offered service as a process (cloud prosumers that provide service mashups).

\section{Overview of the Framework}

Our framework for user-controlled management of policies in multi-tenancy cloud provisionings facilitates the (self-)management (specification and monitoring) of cloud resources in order to optimise usage (the provider perspective) based on monitoring compliancy and to ensure SLA compliance (the user perspective) based on monitoring policies [2]. Our assumption for the service-level solution is that services processes (rather than individual services) are enacted by SaaS providers or users. These are customer policy-enhanced provider processes or customer processes which are customer policy-enhanced. 
A management scalability problem for multi-tenancy applications arises if different users have different requirements $[1,3]$. A configurable policy monitoring technique is the proposed solution $[2,4]$. Customisation of policy management requires a fine-granular multi-tenancy model, where end users can configure and enact (remotely) their specific requirements. Two types of interfaces in cloud applications exist that are internally enacted through service proceses: the upload and management of resources, which can be executed by a provider BPEL process, and functionality that the application uses as a process (the cloud acts as middleware). Policy monitoring is a customer service.

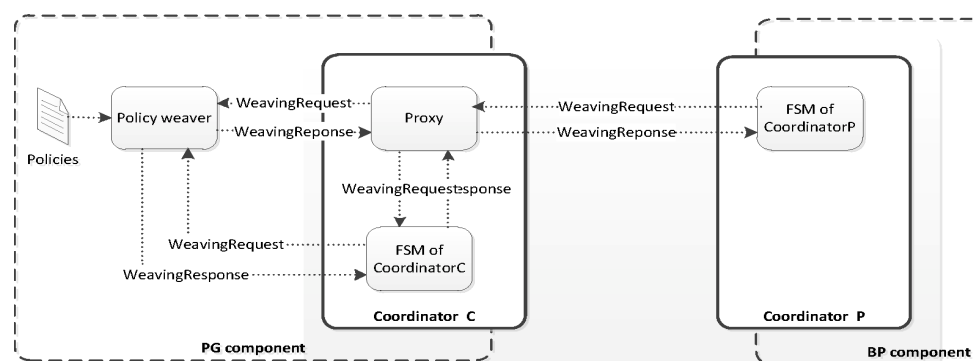

- A policy model allow users to specify dynamic quality and governance requirements. We use rule-based context modelling for policies to capture userspecific requirements and settings [5]. We have implemented user-side components for policy customisation, instrumentation, monitoring and validation.

- A policy coordination and instrumentation software tool that instruments cloud services at process level with the user policies for governance and QoS monitoring $[6,7]$. For the process view, mashups (composition) need to address composition of functionality as well as composition of quality aspects and their respective policy specifications. Monitoring provides feedback for policy customisation - implemented as a listener service for the process.

\section{References}

1. Mietzner, R., Unger, T., Titze, R., Leymann, F. Combining different multi-tenancy patterns in service-oriented applications. In: Intl Enterprise Distributed Object Computing Conf (2009)

2. Wang, M.X., Bandara, K.Y., Pahl, C. Integrated constraint violation handling for dynamic service composition. In: Intl Conf on Services Computing (2009)

3. Erradi, A., Maheshwari, P. and Tosic, V. Policy-driven middleware for selfadaptation of web services compositions. In: Intl Middleware Conf (2006)

4. Zeng, L., Lei, H., Jeng, J.J., Chung, J.Y., Benatallah, B.. Policy-driven exceptionmanagement for composite web services. In: Intl Conf E-Comm Tech (2005)

5. Pahl, C., Zhu, Y. A Semantical Framework for the Orchestration and Choreography of Web Services. International Workshop on Web Languages and Formal Methods WLFM'05. ENTCS (2005)

6. Wu, Y., Doshi, P. Making bpel flexible and adapting in the context of coordination constraints using ws-bpel. In Intl Conf on Services Computing (2008)

7. Pahl, C. A Formal Composition and Interaction Model for a Web Component Platform. In: Workshop on Formal Methods and Component Interaction FMCI02 (2002) 